\title{
Iterative convergence of boundary-volume integral equation method*
}

\author{
Gengxin $\mathrm{Yu}^{*}$ and Liyun $\mathrm{Fu}$ \\ Institute of Geology and Geophysics, Chinese Academy of Sciences, Beijing 100029, China
}

\begin{abstract}
The boundary-volume integral equation numerical technique can be a powerful tool for piecewise heterogeneous media, but it is limited to small problems or low frequencies because of great computational cost. Therefore, a restarted GMRES method is applied to solve large-scale boundary-volume scattering problems in this paper to overcome the computational barrier. The iterative method is firstly applied to responses of dimensionless frequency to a semicircular alluvial valley filled with sediments, compared with the standard Gaussian elimination method. Then the method is tested by a heterogeneous multilayered model to show its applicability. Numerical experiments indicate that the preconditioned GMRES method can significantly improve computational efficiency especially for large Earth models and high frequencies, but with a faster convergence for the left diagonal preconditioning.
\end{abstract}

Key words: boundary-volume integral equation; generalized Lipmann-Schwinger integral equation; GMRES method; diagonal preconditioner

CLC number: P315.01 Document code: A

\section{Introduction}

Boundary-element (BE) methods have been applied to $2 \mathrm{D}$ and $3 \mathrm{D}$ seismic models in exploration geophysics during the past several decades (e.g., Bakamjian, 1992; Bouchon, 1993; Chen and Zhou, 1994; $\mathrm{Fu}$ and $\mathrm{Mu}$, 1994; Dong et al., 1995; Fu, 1996; Liu et al., 2008a, b; Yu et al., 2010). However, these boundary methods are limited by their abilities to handle volume heterogeneities. The volume heterogeneities lead to a coupled boundary-volume interaction that requires an extension of the traditional boundary methods to account for volume heterogeneities. To solve this problem, Fu (2003) incorporated the boundary integral representation into the Lippmann-Schwinger integral equation to model roughly topography in complex near surface areas. The method provides an efficient scheme to synthesize seismograms in piecewise heterogeneous media by the explicit use of boundary continuity conditions across subsurface interfaces. The scattering term associated with volume integrals over volume heterogeneities can

\footnotetext{
* Received 15 September 2011; accepted in revised form 2 October 2011; published 10 October 2011.

* Corresponding author. e-mail: y_g_xin@126.com

(c) The Seismological Society of China and Springer-Verlag Berlin Heidelberg 2011
}

be calculated by accurate Gaussian integration numerical algorithms, which guarantees an optimum seismic modeling for scattering effects by volume heterogeneities with arbitrary complexity.

Although this boundary-volume integral equation numerical technique can be a powerful tool for piecewise heterogeneous media, it is limited to small problems or low frequencies because of great computational cost. The computation becomes extremely prohibitive due to very large size of the resulting matrices to be inverted when the ratio of model dimension to wavelength becomes too large. An efficient alternative is to find a feasible iterative method to overcome the computational barrier. The purpose of this paper is to pursue an efficient iterative solution for boundary-volume scattering problems.

Various iterative methods, such as conjugate gradient square (CGS), the generalized minimal residual method (GMRES), and Bi-conjugate gradient stabilized method (BiCGSTAB), have been widely used to the solution for scattering problems (e.g., Cao and Macaskill, 1995; Zhou and Hong, 2002; Xia et al., 2004; Wei and Liu, 2007a, b). In this paper, a restarted GMRES method is used to solve large-scale boundary-volume scattering problems. The iterative method is firstly validated by dimensionless frequency responses to a semi- 
circular alluvial valley (filled with sediments), compared with the standard Gaussian elimination method. Then the method is tested by a heterogeneous multilayered model to show its applicability. A preconditioning technique is also used to improve convergence rate.

\section{Method}

\subsection{Generalized Lippmann-Schwinger integral equation}

Wave propagation in a large-scale boundary structure with internal volume heterogeneities can be described by a generalized Lipmann-Schwinger integral equation (GLSIE, Fu, 2003). It is formulated as the superposition of incident, boundary-scattering, and volume-scattering waves. The problem to be studied is illustrated in Figure 1. In this model, there are $N$

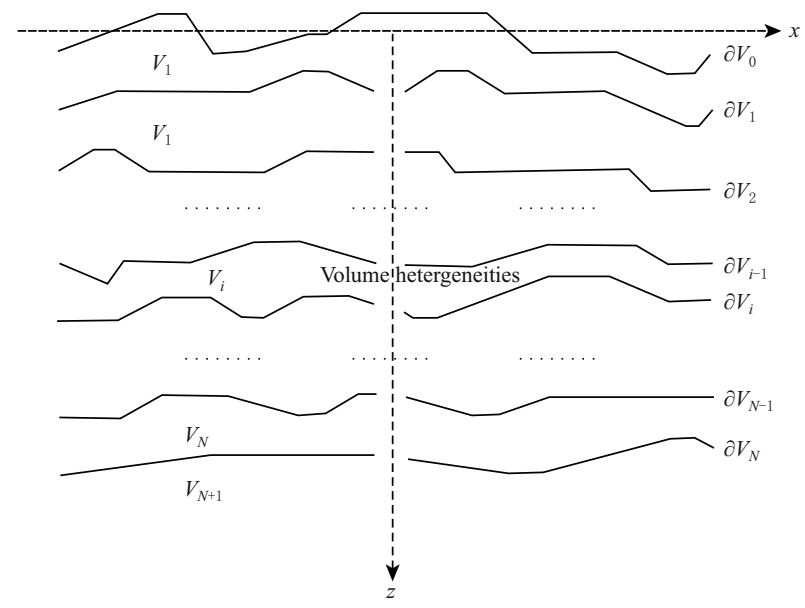

Figure 1 Configuration of the problem considered in this study.

heterogeneous layers $V_{i}(i=1,2, \ldots, N)$ over a half-space, among which the $i$ th layer is bounded by two irregular interfaces $\partial V_{i-1}$ and $\partial V_{i}$. The uppermost interface $\partial V_{0}$ is a free surface, and an arbitrary source is embedded in the $i$ th layer. For simplicity, we restrict the present study to the 2D SH problem (or acoustic problem). The solution domain of the problem for the $i$ th layer is defined as $\bar{V}_{i} \in V_{i}+\partial V_{i-1}+\partial V_{i}$. Seismic response $u(\boldsymbol{r})$ for steady state scalar wave propagation in the $i$ th layer satisfies the following scalar equation

$$
\nabla^{2} u(\boldsymbol{r})+\left[k^{(i)}(\boldsymbol{r})\right]^{2} u(\boldsymbol{r})=-\delta_{s i} s(\boldsymbol{r}, \omega)
$$

$$
\boldsymbol{r} \in \bar{V}_{i} \quad i=1,2, \ldots, N,
$$

where $k^{(i)}(\boldsymbol{r})=\omega / v^{(i)}(\boldsymbol{r}), v^{(i)}(\boldsymbol{r})$ is the velocity distribution of the $i$ th layer, $\delta_{s i}=1$ for $i=s$ and $\delta_{s i}=0$ for $i \neq s, \omega$ is the frequency, $s(\boldsymbol{r}, \omega)$ is the source term and $\nabla^{2}$ is the Laplacian operator. The seismic response $u(\boldsymbol{r})$ also satisfies the following boundary conditions:

1) The traction-free condition on the free surface: $\partial u(\boldsymbol{r}) / \partial n=0$ at $\boldsymbol{r} \in \partial V_{0}$.

2) The continuities of displacement and traction at the interface $\partial V_{i}$,

$$
\left\{\begin{array}{c}
u_{-}^{(i)}(\boldsymbol{r})=u_{+}^{(i)}(\boldsymbol{r}) \\
\frac{\partial u_{-}^{(i)}(\boldsymbol{r})}{\partial n}=\frac{\partial u_{+}^{(i)}(\boldsymbol{r})}{\partial n}
\end{array} \quad \boldsymbol{r} \in \partial V_{i},\right.
$$

where '_' denotes the top side of $\partial V_{i}$ toward $V_{i}$ and '+' denotes the underside of $\partial V_{i}$ toward $\partial V_{i+1}$.

3) The radiation boundary condition imposed on the far-field behavior at infinity.

$$
\left\{\begin{array}{c}
\lim _{|r| \rightarrow \infty} u(\boldsymbol{r})=0 \\
\lim _{|r| \rightarrow \infty} \frac{\partial u(\boldsymbol{r})}{\partial r}=0
\end{array} .\right.
$$

To explicitly use the boundary condition defined by equation (2) in the solution of the problem, we transform equation (1) into an integral equation in terms of boundary and volume integrals of the solution domain. The scattering sources in the solution domain $\bar{V}_{i}$ include the top boundary $\partial V_{i-1}$, the bottom boundary $\partial V_{i}$, the seismic source and the volume heterogeneities in $V_{i}$. According to integral equation theory (Pao and Varatharajulu, 1976), the total seismic response $u(\boldsymbol{r})$ at a location $\boldsymbol{r} \in \bar{V}_{i}$ is composed of

$$
u(\boldsymbol{r})=u^{0}(\boldsymbol{r})+u_{1}^{s}(\boldsymbol{r})+u_{2}^{s}(\boldsymbol{r})+u_{3}^{s}(\boldsymbol{r}) .
$$

$u^{0}(\boldsymbol{r})$ is the incident field in the background medium and can be represented as

$$
\begin{gathered}
u^{0}(\boldsymbol{r})=\int_{V_{i}} s^{(i)}\left(\boldsymbol{r}^{\prime}, \omega\right) G^{(i)}\left(\boldsymbol{r}, \boldsymbol{r}^{\prime}\right) \mathrm{d} \boldsymbol{r}^{\prime}= \\
\delta_{s i} S(\omega) G^{(i)}\left(\boldsymbol{r}, \boldsymbol{r}_{0}\right)
\end{gathered},
$$

where $G^{(i)}\left(\boldsymbol{r}, \boldsymbol{r}_{0}\right)$ is the Green's function. $u_{1}^{s}(\boldsymbol{r})$ is the boundary field scattered by the top boundary $\partial V_{i-1}$ and satisfies the following boundary integral equation:

$$
u_{1}^{s}(\boldsymbol{r})=\int_{\partial V_{i-1}}\left[G^{(i)}\left(\boldsymbol{r}, \boldsymbol{r}^{\prime}\right) t^{(i-1)}\left(\boldsymbol{r}^{\prime}\right)-u^{(i-1)}\left(\boldsymbol{r}^{\prime}\right) \frac{\partial G^{(i)}\left(\boldsymbol{r}, \boldsymbol{r}^{\prime}\right)}{\partial n}\right] \mathrm{d} \boldsymbol{r}^{\prime},
$$


where $u^{(i-1)}(\boldsymbol{r})$ is the displacement on $\partial V_{i-1}$, $t^{(i-1)}(\boldsymbol{r})=\partial u^{(i-1)}(\boldsymbol{r}) / \partial n$ is the normal gradient of the displacement on $\partial V_{i-1}$. Similarly, $u_{2}^{s}(\boldsymbol{r})$ is the boundary field scattered by the bottom boundary $\partial V_{i}$ and satisfies the following boundary integral equation:

$$
u_{2}^{s}(\boldsymbol{r})=\int_{\partial V_{i}}\left[G^{(i)}\left(\boldsymbol{r}, \boldsymbol{r}^{\prime}\right) t^{(i)}\left(\boldsymbol{r}^{\prime}\right)-u^{(i)}\left(\boldsymbol{r}^{\prime}\right) \frac{\partial G^{(i)}\left(\boldsymbol{r}, \boldsymbol{r}^{\prime}\right)}{\partial n}\right] \mathrm{d} \boldsymbol{r}^{\prime}
$$

$u_{3}^{s}(\boldsymbol{r})$ is the volume field scattered by the volume heterogeneities in $V_{i}$ and satisfies the following LippmannSchwinger integral equation:

$$
u_{3}^{s}(\boldsymbol{r})=\left[k_{0}^{(i)}\right]^{2} \int_{V_{i}} O^{(i)}\left(\boldsymbol{r}^{\prime}\right) w^{(i)}\left(\boldsymbol{r}^{\prime}\right) G^{(i)}\left(\boldsymbol{r}, \boldsymbol{r}^{\prime}\right) d \boldsymbol{r}^{\prime},
$$

where $k_{0}^{(i)}=\omega / v_{0}^{(i)}$ is the background wavenumber, $v_{0}^{(i)}$ is the background velocity of the ith layer, the relative slowness perturbation $O^{(i)}(\boldsymbol{r})=\left[v_{0}^{(i)} / v^{(i)}(\boldsymbol{r})\right]^{2}-1$, and $w^{(i)}(\boldsymbol{r})$ is the displacement inside $V_{i}$.

Substituting equations (5-8) into equation (4) and considering the "boundary naturalization" of the integral equations, that is, a limit analysis when the "observation point" $\boldsymbol{r}=(x, z)$ approaches the boundary $\partial V_{i}$ and tends to coincide with the "scattering point" $\boldsymbol{r}^{\prime}=\left(x^{\prime}, z^{\prime}\right)$ when $\boldsymbol{r}^{\prime} \in \partial V_{i}$, we obtain the GLSIE:

$$
\begin{array}{r}
\int_{\partial V_{i-1}}\left[G^{(i)}\left(\boldsymbol{r}, \boldsymbol{r}^{\prime}\right) t^{(i-1)}\left(\boldsymbol{r}^{\prime}\right)-u^{(i-1)}\left(\boldsymbol{r}^{\prime}\right) \frac{\partial G^{(i)}\left(\boldsymbol{r}, \boldsymbol{r}^{\prime}\right)}{\partial n}\right] \mathrm{d} \boldsymbol{r}^{\prime}+\int_{\partial V_{i}}\left[G^{(i)}\left(\boldsymbol{r}, \boldsymbol{r}^{\prime}\right) t^{(i)}\left(\boldsymbol{r}^{\prime}\right)-u^{(i)}\left(\boldsymbol{r}^{\prime}\right) \frac{\partial G^{(i)}\left(\boldsymbol{r}, \boldsymbol{r}^{\prime}\right)}{\partial n}\right] \mathrm{d} \boldsymbol{r}^{\prime}+ \\
\left(k_{0}^{(i)}\right)^{2} \int_{V_{i}} O^{(i)}\left(\boldsymbol{r}^{\prime}\right) w^{(i)}\left(\boldsymbol{r}^{\prime}\right) G^{(i)}\left(\boldsymbol{r}, \boldsymbol{r}^{\prime}\right) \mathrm{d} \boldsymbol{r}^{\prime}+\delta_{s i} S(\omega) G^{(i)}\left(\boldsymbol{r}, \boldsymbol{r}_{0}\right)= \begin{cases}w^{(i)}(\boldsymbol{r}) & \boldsymbol{r} \in V_{i} \\
C^{(i-1)}(\boldsymbol{r}) u^{(i-1)}(\boldsymbol{r}) & \boldsymbol{r} \in \partial V_{i-1} \\
C^{(i)}(\boldsymbol{r}) u^{(i)}(\boldsymbol{r}) & \boldsymbol{r} \in \partial V_{i} \\
0 & \boldsymbol{r} \notin \bar{V}_{i}\end{cases}
\end{array}
$$

where the coefficients $C(\boldsymbol{r})$ generally depend on the local geometry at $\boldsymbol{r}$. For piecewise homogeneous media [i.e. $O^{(i)}(\boldsymbol{r})=0$ ], $u_{3}^{s}(\boldsymbol{r})=0$ and equation (4) reduces to a standard boundary integral equation.

The causal Green's function is defined everywhere in the free space, relating an observation point $\boldsymbol{r}$ to a scattering point $\boldsymbol{r}^{\prime}$. It satisfies the homogeneous Helmholtz equation in the reference medium:

$$
\begin{gathered}
\nabla^{2} G^{(i)}\left(\boldsymbol{r}, \boldsymbol{r}^{\prime}\right)+\left[k_{0}^{(i)}\right]^{2} G^{(i)}\left(\boldsymbol{r}, \boldsymbol{r}^{\prime}\right)= \\
-\delta\left(\boldsymbol{r}-\boldsymbol{r}^{\prime}\right) \quad \boldsymbol{r}, \boldsymbol{r}^{\prime} \in V_{i} .
\end{gathered}
$$

For 2-D problems, the Green's function is given by (Abramowitz and Stegun, 1968)

$$
G^{(i)}\left(\boldsymbol{r}, \boldsymbol{r}^{\prime}\right)=\frac{i}{4} \mathrm{H}_{0}^{(1)}\left[k_{0}^{(i]}\left|\boldsymbol{r}^{\prime}-\boldsymbol{r}\right|\right],
$$

where $i=\sqrt{-1}$ and $\mathrm{H}_{0}^{(1)}$ denotes the Hankel function of the first kind and of zeroth order. These integral equations naturally satisfy Sommerfeld non-reflecting and decay boundary conditions defined by equation (3).

\subsection{Numerical discretization of boundary- volume integral equations}

The collocation method has been widely used for numerical solutions of all types of integral equations. The numerical solution of equation (9) by the collocation method involves several steps. Each boundary $\partial V_{i}$ is divided into $L^{(i)}$ boundary elements denoted by $\Gamma_{e}\left(e=1,2, \ldots, L^{(i)}\right)$ and each volume $V_{i}$ into $M^{(i)}$ finite elements denoted by $\Omega_{e}\left(e=1,2, \ldots, M^{(i)}\right)$. The total node number of $\bar{V}_{i}$ is $N^{(i)}$. In the collocation method, interpolation shape functions $\phi$ are used so that the variables $(\boldsymbol{r}, u, t$ and $w)$ are approximated by the linear combination of their nodal values over an element $\Gamma_{e}$ or $\Omega_{e}$ defined geometrically between the node $I_{1}$ and $I_{2}$, for instance,

$$
u^{(i)}(\xi)=\sum_{l=I_{1}}^{I_{2}} u^{(i)}\left(\boldsymbol{r}_{l}\right) \phi_{l}(\xi),
$$

where $\xi$ denotes the local coordinate of an element. The integrals in equation (9) can be computed over each element respectively as 


$$
\begin{gathered}
h_{j k}^{(i, 1)}=\sum_{e=1}^{L^{(i-1)}} \sum_{l=I_{1}}^{I_{2}}\left[\int_{\Gamma_{e}} \frac{\partial}{\partial n} G^{(i)}\left[\boldsymbol{r}_{j}, \boldsymbol{r}^{\prime}(\xi)\right] \phi_{l}(\xi) \mathrm{d} \boldsymbol{r}^{\prime}(\xi)\right] \delta_{l k}+C^{(i-1)}\left(\boldsymbol{r}_{j}\right) \delta_{j k}, \\
g_{j k}^{(i, 1)}=\sum_{e=1}^{L^{(i-1)}} \sum_{l=I_{1}}^{I_{2}}\left[\int_{\Gamma_{e}} G^{(i)}\left[\boldsymbol{r}_{j}, \boldsymbol{r}^{\prime}(\xi)\right] \phi_{l}(\xi) \mathrm{d} \boldsymbol{r}^{\prime}(\xi)\right] \delta_{l k}, \\
h_{j k}^{(i, 2)}=\sum_{e=1}^{L^{(i)}} \sum_{l=I_{1}}^{I_{2}}\left[\int_{\Gamma_{e}} \frac{\partial}{\partial n} G^{(i)}\left[\boldsymbol{r}_{j}, \boldsymbol{r}^{\prime}(\xi)\right] \phi_{l}(\xi) \mathrm{d} \boldsymbol{r}^{\prime}(\xi)\right] \delta_{l k}+C^{(i)}\left(\boldsymbol{r}_{j}\right) \delta_{j k}, \\
g_{j k}^{(i, 2)}=\sum_{e=1}^{L^{(i)}} \sum_{l=I_{1}}^{I_{2}}\left[\int_{\Gamma_{e}} G^{(i)}\left[\boldsymbol{r}_{j}, \boldsymbol{r}^{\prime}(\xi)\right] \phi_{l}(\xi) \mathrm{d} \boldsymbol{r}^{\prime}(\xi)\right] \delta_{l k}, \\
K_{j k}^{(i)}=\sum_{e=1}^{M^{(i)}} \sum_{l=I_{1}}^{I_{2}}\left\{\left[k_{0}^{(i)}\right]^{2} \int_{\Omega_{e}} O^{(i)}\left[\boldsymbol{r}^{\prime}(\xi)\right] G^{(i)}\left[\boldsymbol{r}_{j}, \boldsymbol{r}^{\prime}(\xi)\right] \phi_{l}(\xi) \mathrm{d} \boldsymbol{r}^{\prime}(\xi)\right\} \delta_{l k}-\delta_{j k}
\end{gathered}
$$

where $\delta_{l k}$ and $\delta_{j k}$ are the Kronecker delta functions. The Gaussian integration algorithm is used to numerically evaluate these integrals (Xu, 1995). Equation (9) can be written in an operator form

$$
\begin{aligned}
\boldsymbol{H}^{(i, 1)} u^{(i-1)}\left(\boldsymbol{r}_{j}\right)- & \boldsymbol{G}^{(i, 1)} t^{(i-1)}\left(\boldsymbol{r}_{j}\right)+\boldsymbol{H}^{(i, 2)} u^{(i)}\left(\boldsymbol{r}_{j}\right)- \\
\boldsymbol{G}^{(i, 2)} t^{(i)}\left(\boldsymbol{r}_{j}\right) & =\boldsymbol{K}^{(i)} w^{(i)}\left(\boldsymbol{r}_{j}\right)+\delta_{s i} f\left(\boldsymbol{r}_{j}\right) \\
j & =1,2, \ldots, N^{(i)},
\end{aligned}
$$

where $f$ is the incident field $\boldsymbol{H}^{(i, 1)}$ and $\boldsymbol{G}^{(i, 1)}$ are the top-boundary coefficient matrices composed of $h_{j k}^{(i, 1)}$ and $g_{j k}^{(i, 1)}, \boldsymbol{H}^{(i, 2)}$ and $\boldsymbol{G}^{(i, 2)}$ are the bottom-boundary coefficient matrices consisting in $h_{j k}^{(i, 2)}$ and $g_{j k}^{(i, 2)}$, and $\boldsymbol{K}^{(i)}$ is the perturbation-domain coefficient matrix composed of $K_{j k}^{(i)}$.

Equation (18) can be further compacted as a matrix equation for $j=1,2, \ldots, N^{(i)}$

$$
\boldsymbol{A}^{(i, 1)} \boldsymbol{m}^{(i-1)}+\boldsymbol{A}^{(i, 2)} \boldsymbol{m}^{(i)}=\boldsymbol{K}^{(i)} \boldsymbol{w}^{(i)}+\delta_{s i} \boldsymbol{f}
$$

where we define the boundary coefficient matrices $\boldsymbol{A}^{(i, 1)}=\left[\boldsymbol{H}^{(i, 1)},-\boldsymbol{G}^{(i, 1)}\right]$ and $\boldsymbol{A}^{(i, 2)}=\left[\boldsymbol{H}^{(i, 2)},-\boldsymbol{G}^{(i, 2)}\right]$, and the unknown boundary displacement-traction vector $\boldsymbol{m}^{i}=\left[u^{(i)}, t^{(i)}\right]$. Solving the linear system of equations (19) results in seismic responses $u(\boldsymbol{r})$ for all nodes in the medium. Since a considerable amount of matrix operations are involved and the matrix for each frequency component must be inverted, the GLSIE method is computationally intensive at high frequencies. Therefore, an efficient iterative method is used here to solve equation (19).

\subsection{The GMRES method}

The generalized minimum residual (GMRES) method was proposed by Saad and Schultz (1986) in order to solve large, sparse and non Hermitian linear systems. GMRES belongs to the class of Krylov based iterative methods. It relies on the Arnoldi process, and the approximate solution has the property of minimizing at every step the norm of the residual vector over the Krylov subspace (Ma, 2005). However, the Arnoldi orthogonalization process requires a large amount of memory especially when the subspace grows. Therefore a restarted technique is generally used to cope with this memory drawback.

The boundary-volume integral equation (19) can also be written as

$$
\boldsymbol{A} \psi=f,
$$

where $\boldsymbol{A} \in R^{n \times n}$ and $\psi, f \in R^{n}$. Suppose $\psi_{0}$ is an initial guess to the solution, and $\boldsymbol{r}_{0}=f-\boldsymbol{A} \psi_{0}$ is the initial residual vector. The GMRES method builds an approximate solution of the form

$$
\psi_{m}=\psi_{0}+\boldsymbol{V}_{m} y_{m},
$$

where $\boldsymbol{V}_{m}$ is an orthonormal basis for the Krylov subspace of dimension $m$ defined by

$$
K_{m}=\operatorname{Span}\left\{\boldsymbol{r}_{0}, \boldsymbol{A} \boldsymbol{r}_{0}, \cdots, \boldsymbol{A}^{m-1} \boldsymbol{r}_{0}\right\},
$$

and where $y_{m} \in R^{m}$. The vector $\boldsymbol{y}$ is determined so that the 2-norm of the residual $r_{m}=f-A \psi_{m}$ is minimal over $K_{m}$. The basis $\boldsymbol{V}_{m}$ for the Krylov subspace $K_{m}$ is obtained via the well-known Arnoldi process (Ma, 2005). The orthogonal projection of $\boldsymbol{A}$ onto $K_{m}$ results in an 
upper Hessenberg matrix $\boldsymbol{H}_{m}=\boldsymbol{V}_{m}^{\mathrm{T}} \boldsymbol{A} \boldsymbol{V}_{m}$ of order $m$. The Arnoldi process satisfies the relationship

$$
\boldsymbol{A} \boldsymbol{V}_{m}=\boldsymbol{V}_{m} \boldsymbol{H}_{m}+h_{m+1, m} v_{m+1} \boldsymbol{e}_{m}^{\mathrm{T}}
$$

where $\quad \boldsymbol{V}_{m}=\left(v_{1}, v_{2}, \cdots, v_{m}\right), \quad h_{m+1, m}=\left\|\tilde{v}_{m+1}\right\|_{2}$, $v_{m+1}=\tilde{v}_{m+1} / h_{m+1, m}, \quad \tilde{v}_{m+1}=\boldsymbol{A} v_{m}-\sum_{i=1}^{m} h_{i, m} v_{i}, \quad h_{i, m}$ $=\left(\boldsymbol{A} v_{m}, v_{i}\right)$, and $\boldsymbol{e}_{m}$ is the $m$ th canonical basis vector. Equation (23) can be rewritten as

$$
\boldsymbol{A} \boldsymbol{V}_{m}=\boldsymbol{V}_{m+1} \overline{\boldsymbol{H}}_{m}
$$

where $\overline{\boldsymbol{H}}_{m}=\left[\begin{array}{c}H_{m} \\ 0 \ldots 0 \quad h_{m+1, m}\end{array}\right]$ is an $(m+1) \times m$ upper Hessenberg matrix.

Let $v_{1}=r_{0} / \beta$ where $\beta=\left\|r_{0}\right\|$. The residual asso- ciated with the approximate solution verifies

$$
\begin{gathered}
r_{m}=f-\boldsymbol{A} \psi_{m}=f-\boldsymbol{A}\left(\psi_{0}+\boldsymbol{V}_{m} y_{m}\right)=r_{0}-\boldsymbol{A} \boldsymbol{V}_{m} y_{m}= \\
\beta v_{1}-\boldsymbol{V}_{m+1} \overline{\boldsymbol{H}}_{m} y_{m}=\boldsymbol{V}_{m+1}\left(\beta e_{1}-\overline{\boldsymbol{H}}_{m} y_{m}\right) .
\end{gathered}
$$

Since $\boldsymbol{V}_{m}$ is a matrix with orthonormal columns, the residual norm $\left\|r_{m}\right\|=\left\|\beta e_{1}-\overline{\boldsymbol{H}}_{m} y_{m}\right\|$ is minimal when $y_{m}$ solves the linear least-squares problem

$$
\min _{y_{m} \in R^{m}}\left\|\beta e_{1}-\bar{H}_{m} y_{m}\right\|
$$

The following is a flowchart of the restarted GMRES algorithm (Figure 2). It is important to choose an appropriate restart parameter $m$ as the choice can significantly affect the convergence rate. The restart parameter $m$ is generally chosen small relative to $n$ to keep storage and computation requirements reasonable.

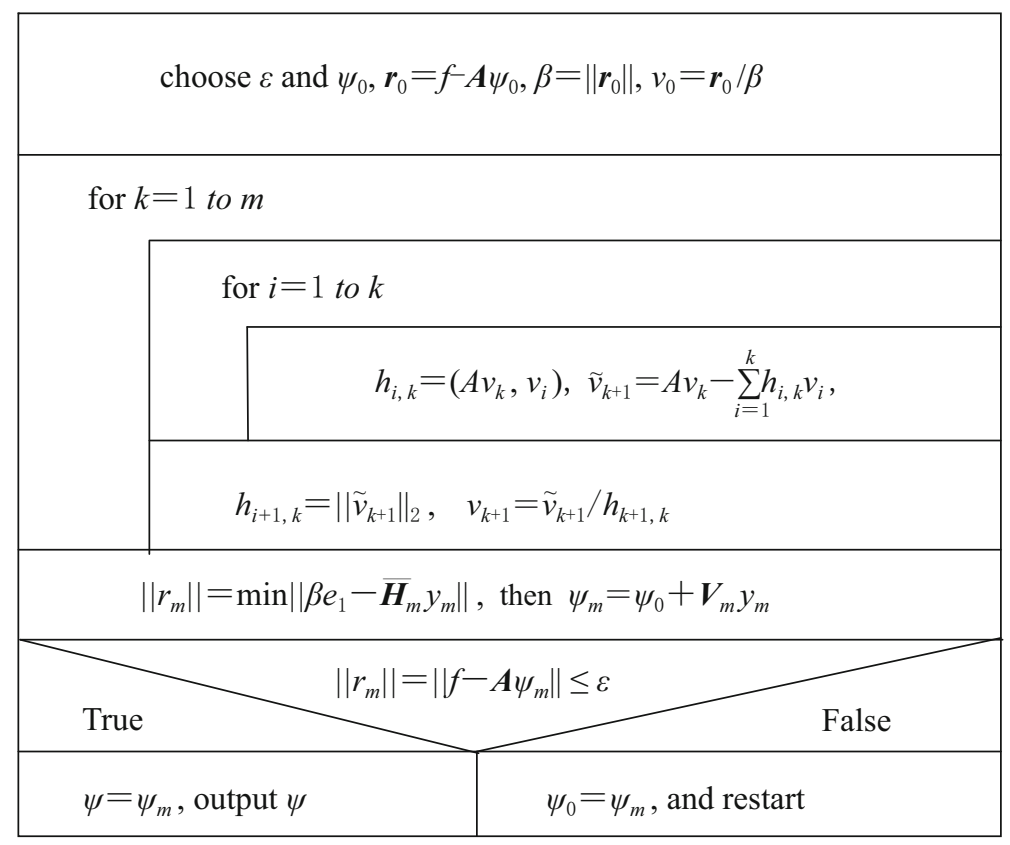

Figure 2 A flow chart of the restarted GMRES algorithm.

Preconditioning techniques are often used to improve the performance and reliability of Krylov subspace methods. The term preconditioning refers to transforming the system $\boldsymbol{A}$ into another system with more favorable properties for iterative solution. In general, a good preconditioner should meet the following requirements: (1) The preconditioned system should be easy to solve; (2) The preconditioner should be cheap to construct and apply. With a good preconditioner, the computing time for the preconditioned iteration should be significantly less than that for the unpreconditioned one. If the preconditioner $\boldsymbol{M}$ is written in the form $\boldsymbol{M}=\boldsymbol{M}_{1} \cdot \boldsymbol{M}_{2}$, we actually solve the linear system

$$
\boldsymbol{M}_{1}^{-1} \boldsymbol{A} \boldsymbol{M}_{2}^{-1} \psi^{\prime}=\boldsymbol{M}_{1}^{-1} f
$$

where $\psi^{\prime}=\boldsymbol{M}_{2}^{-1} \psi$. Note that $\boldsymbol{M}_{2}=\boldsymbol{I}$ for left preconditioning, and $\boldsymbol{M}_{1}=\boldsymbol{I}$ for right preconditioning. In this paper both left and right diagonal preconditioning techniques are applied to accelerate convergence and compared with each other. 


\section{Numerical tests}

The iterative method is tested to show its effectiveness by modeling a semicircular alluvial valley (filled with sediments) and a heterogeneous near surface layered model with flat topography, compared with the standard Gaussian elimination method. Numerical modeling is implemented in the frequency domain. A variable element dimension technique was adopted in the program implementation $(\mathrm{Fu}, 1996)$ to improve computation speed because sampling at three elements per wavelength is sufficient to ensure the accuracy of the results (Campillo, 1987). Because the wavelength is the function of frequencies and velocities, the dimension of boundary elements for each subregion in a model is computed according to the medium velocity and the computational frequency. The model is then automatically discretized in terms of updated element dimensions for each computational frequency.

Shown in Figure $3 \mathrm{a}$ is a complex fault model with six subregions $\Omega_{i}(i=1,2, \ldots, 6)$ with the wave velocities $(\mathrm{km} / \mathrm{s})$ indicated in the figure. The dimensions of the model are $3800 \mathrm{~m}$ horizontally and $1500 \mathrm{~m}$ vertically. The source is a minimum- phase Gauss wavelet with a central frequency of 15 $\mathrm{Hz}$. The stacked sections are calculated in the frequency range $0-40 \mathrm{~Hz}$, with receivers along the free surface. Figure $3 \mathrm{~b}$ shows the stacked section calculated using the finite-difference method made by Seismic Un* $\mathrm{x}$ (http://www.cwp.mines.edu/cwpcodes/). In comparison with our result (Figure 3c) given by equation (9), we see a perfect agreement between them but a more distinct description of the fault interface for the latter. The above comparison confirms the validity of my formulations and computation program.

The semicircular hetergeneous valley of radius $a$ is shown in Figure 4. The frequency responses along the horizontal topography to a vertical incident wave are computed for the dimensionless frequency $\eta=1.0$. The dimensionless frequency is defined as $\eta=2 a / \lambda$, where $\lambda$ is the wavelength of incident waves. The shear wave velocity inside the valley is $1500 \mathrm{~m} / \mathrm{s}$ in the background medium of $3000 \mathrm{~m} / \mathrm{s}$. For valley velocity perturbation of 0 percent, the dimension of the resulting matrix is $309 \times 309$. For valley velocity perturbations of $10-25$ percent, the dimension of the resulting matrix is $912 \times 912$. Here the stopping criterion $\varepsilon$ is $1 \times 10^{5}$, and the restart parameter $m$ is 34 .

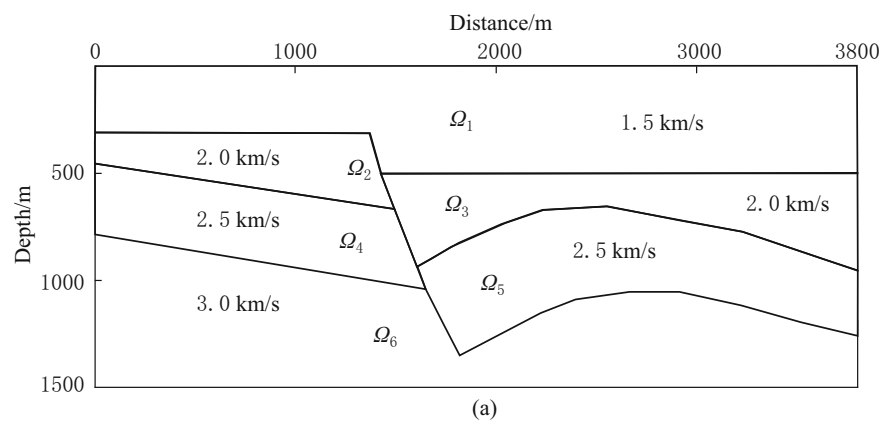

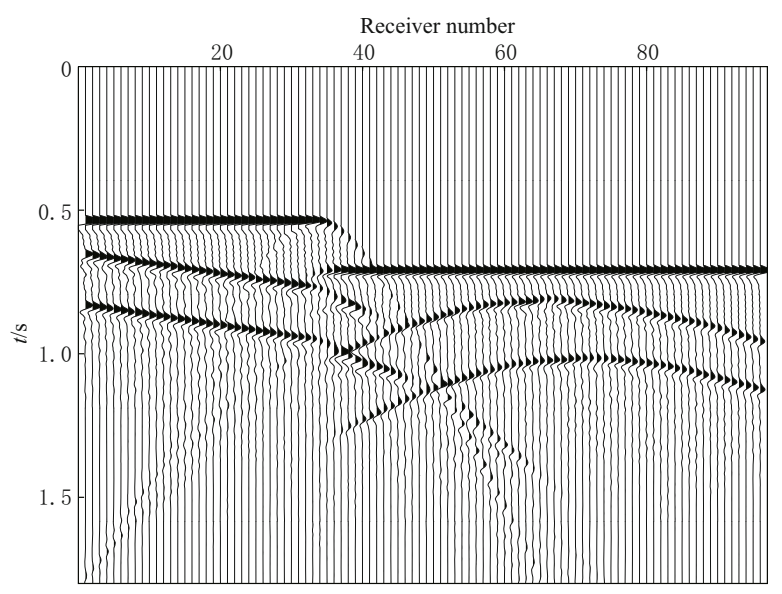

(b)

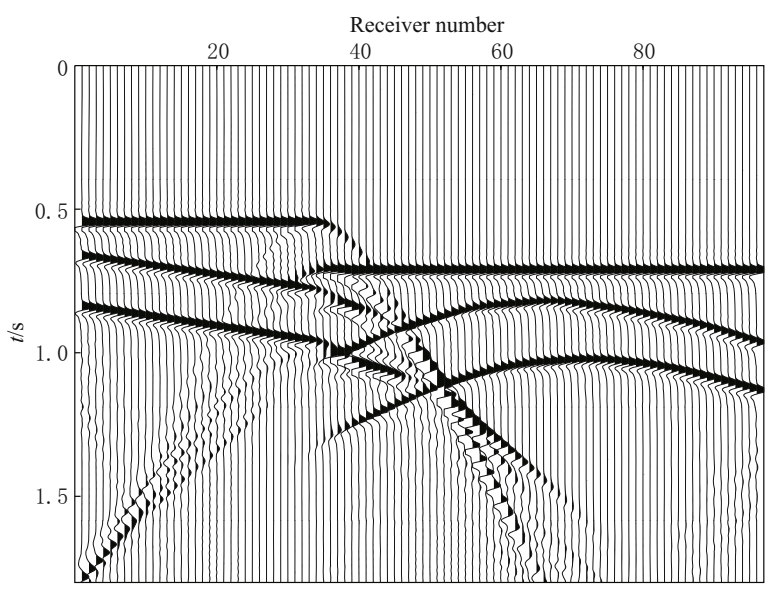

(c)

Figure 3 The geometry of a complex fault model (a) with six subregions $\Omega_{i}(i=1,2, \ldots, 6)$, and the seismic sections for the complex fault model by the finite-difference method (b). The result in (c) is given by equation (9). 
Figures 5 and 6 show the comparison of convergence of different valley velocity perturbations by nonpreconditioned, left and right diagonal preconditioned GMRES (m) method. The experiments indicate that the preconditioned methods have a considerable improvement over non-preconditioned method, and the left diagonal preconditioned method is slightly better than the right diagonal preconditioned one. These above results are further confirmed by the comparison between the GMRES(m) methods and the Gaussian elimination method in Tables 1 and 2. Figures 7 and 8 show the comparison of iterative results by the left preconditioned

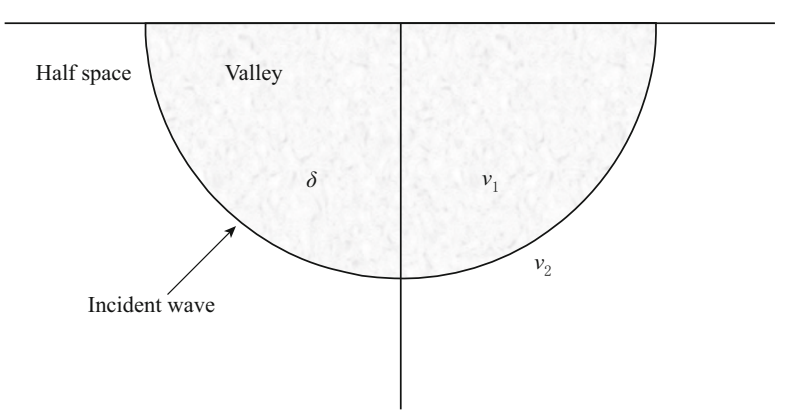

Figure 4 Geometry of a semicircular valley with the radius of $a$ and the wave velocity $\nu_{1}$ in the surrounding homogeneous half-space with the wave velocity $\nu_{2}$.

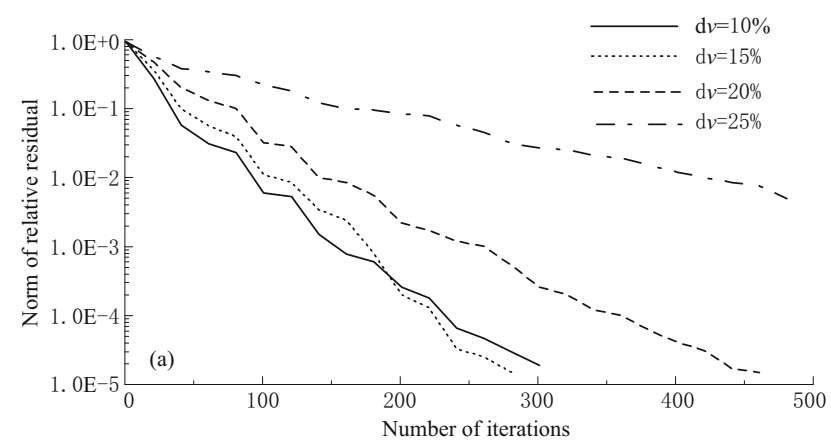

GMRES $(\mathrm{m})$ method with random velocity perturbations of 0 and 15 percent, respectively. It can be seen that the convergence is still fast when the matrix dimension increases from 309 to 912.

Figure 9 shows a heterogeneous multilayered model with randomly velocity perturbation in the near surface layer and the wave velocity indicated in the figure. The computations for this example are performed with frequency range $0-40 \mathrm{~Hz}$ and the source at depth $20 \mathrm{~m}$ below the free surface. The dimensions of the model are $3000 \mathrm{~m}$ horizontally and $1000 \mathrm{~m}$ vertically. The source is a minimum-phase Gauss wavelet with a central

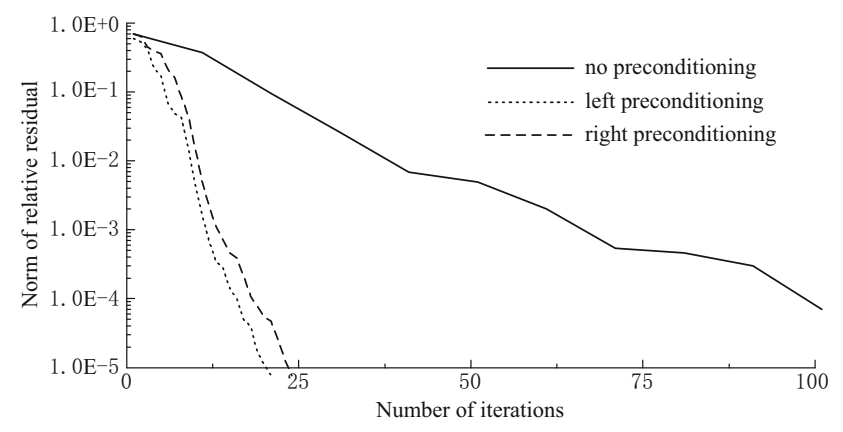

Figure 5 Comparison of convergence by nonpreconditioned (solid line), left (dotted line) and right (dashed line) diagonal preconditioned GMRES (m) method for valley velocity perturbation of 0 percent.

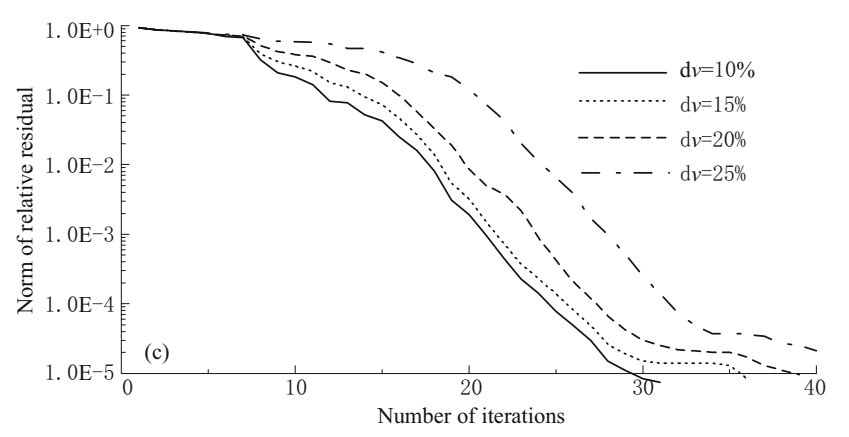

Figure 6 Comparison of convergence for different valley velocity perturbations of 10-25 percent by non-preconditioned (a), left (b) and right (c) diagonal preconditioned GMRES (m) method. 
Table 1 Comparison of different algorithms for the semicircular valley with random velocity perturbation of 0 percent

\begin{tabular}{ccccc}
\hline Algorithm & $\begin{array}{c}\text { Gaussian elimination } \\
\text { method }\end{array}$ & $\begin{array}{c}\text { Non-preconditioned } \\
\text { GMRES }(\mathrm{m})\end{array}$ & $\begin{array}{c}\text { Left preconditioned } \\
\text { GMRES }(\mathrm{m})\end{array}$ & $\begin{array}{c}\text { Right preconditioned } \\
\text { GMRES(m) }\end{array}$ \\
\hline Number of iterations & $/$ & 159 & 21 & 24 \\
CPU time/s & 2.3 & 2.23 & 0.28 & 0.79 \\
\hline
\end{tabular}

Table 2 Comparison of different algorithms for the semicircular valley with random velocity perturbation of 15 percent

\begin{tabular}{ccccc}
\hline Algorithm & $\begin{array}{c}\text { Gaussian elimination } \\
\text { method }\end{array}$ & $\begin{array}{c}\text { Non-preconditioned } \\
\text { GMRES }(\mathrm{m})\end{array}$ & $\begin{array}{c}\text { Left preconditioned } \\
\text { GMRES }(\mathrm{m})\end{array}$ & $\begin{array}{c}\text { Right preconditioned } \\
\text { GMRES(m) }\end{array}$ \\
\hline Number of iterations & $/$ & 296 & 28 & 37 \\
CPU time/s & 11 & 6.23 & 1.46 & 3.09 \\
\hline
\end{tabular}

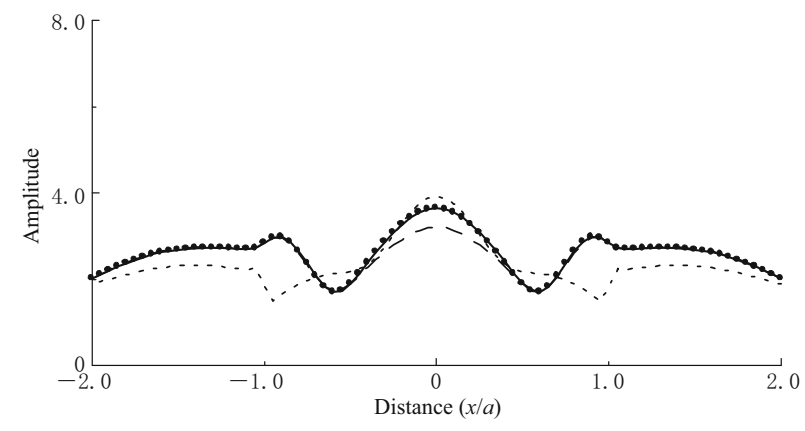

Figure 7 Comparison between the accurate solution of equation (9) (solid line) and the iterative solutions of the fifth (dotted line), the tenth (dashed line) and the fifteenth (dots) iterations for random velocity perturbation of 0 percent. Frequency responses are computed resulting from vertical incident $\mathrm{SH}$ wave with $\eta=1.0$ to the semicircular valley.

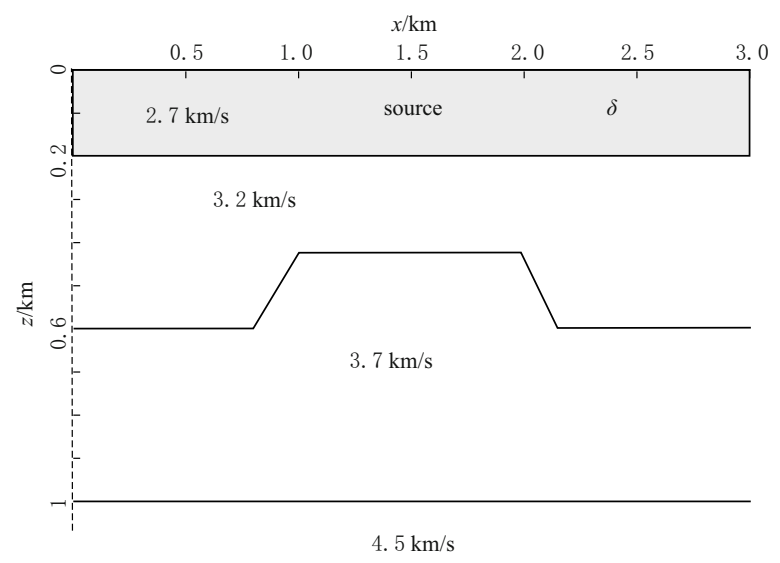

Figure 9 A heterogeneous near-surface layer with flat topography ( $\delta$ is the velocity perturbation).

frequency of $15 \mathrm{~Hz}$. For 0 percent velocity perturbation in the near-surface layer at frequency of $40 \mathrm{~Hz}$, the dimension of the resulting matrix is $1535 \times 1535$. For 15 percent velocity perturbation, the dimension of the

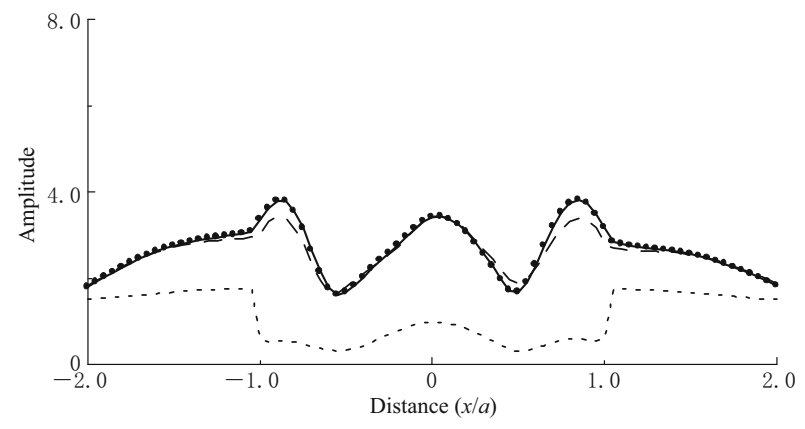

Figure 8 Comparison between the accurate solution of equation (9) (solid line) and the iterative solutions of the fifth (dotted line), the fifteenth (dashed line) and the twenty-fifth (dots) iterations for random velocity perturbation of 15 percent. Frequency responses are computed resulting from vertical incident $\mathrm{SH}$ wave with $\eta=1.0$ to the semicircular valley.

resulting matrix is $4338 \times 4338$. Here the stopping criterion is $1 \times 10^{5}$, and the restart parameter $m$ is 40 .

The comparisons in Figure 10 indicate that the preconditioned GMRES(m) methods perform still well for such larger matrix computations, whereas the nonpreconditioned GMRES(m) method doesn't work. The results in Tables 3 and 4 show that the preconditioned GMRES(m) methods improve the computational efficiency greatly by comparison with the Gaussian elimination method. We also evaluate the accuracies of iterative solutions by the comparison of the waveform recording in Figure 11. The result of the preconditioned GMRES(m) method shows an excellent agreement with that of the Gaussian elimination method.

\section{Conclusions}

In this paper, the restarted GMRES method is used to solve large boundary-volume integral equations. The iterative method is validated by dimensionless frequency responses to a semicircular alluvial valley (filled 

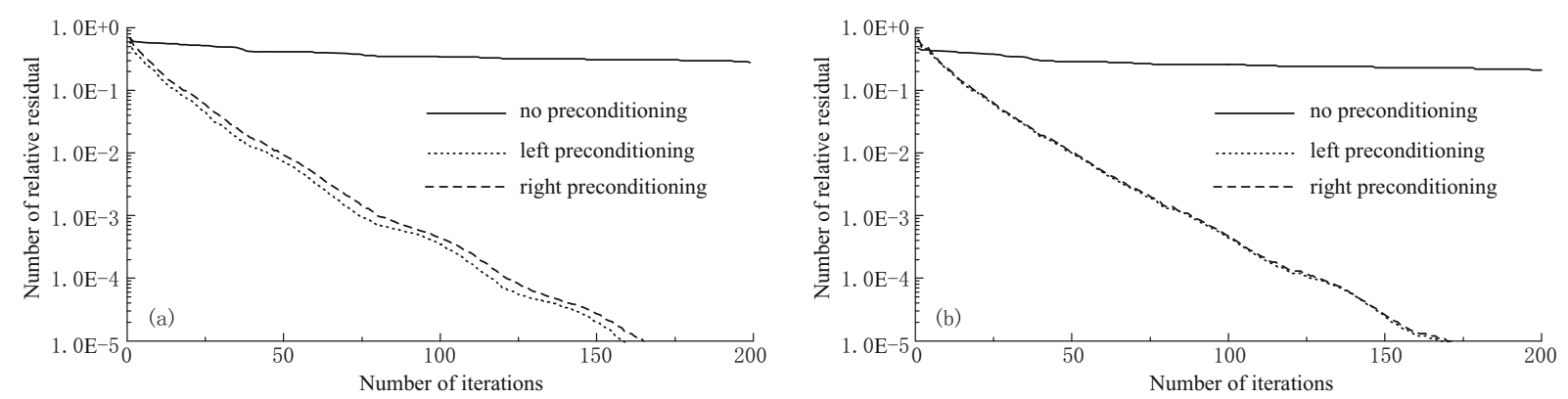

Figure 10 Comparison of convergence by non-preconditioned (solid line), left (dotted line) and right (dashed line) diagonal preconditioned GMRES(m) method for random velocity perturbations of 0 (a) and 15 (b) percent in the near-surface layer, respectively.

Table 3 Comparison of different algorithms for the multilayered model with randomly 0 percent velocity perturbation in the near-surface layer

\begin{tabular}{ccccc}
\hline Algorithm & $\begin{array}{c}\text { Gaussian elimination } \\
\text { method }\end{array}$ & $\begin{array}{c}\text { Non-preconditioned } \\
\text { GMRES }(\mathrm{m})\end{array}$ & $\begin{array}{c}\text { Left preconditioned } \\
\text { GMRES }(\mathrm{m})\end{array}$ & $\begin{array}{c}\text { Right preconditioned } \\
\text { GMRES(m) }\end{array}$ \\
\hline Number of iterations & $/$ & $>1000$ & 159 & 165 \\
CPU time/s & 56.63 & $/$ & 26.54 & 28.17 \\
\hline
\end{tabular}

Table 4 Comparison of different algorithms for the multilayered model with randomly 15 percent velocity perturbation in the near-surface layer

\begin{tabular}{ccccc}
\hline Algorithm & $\begin{array}{c}\text { Gaussian elimination } \\
\text { method }\end{array}$ & $\begin{array}{c}\text { Non-preconditioned } \\
\text { GMRES }(\mathrm{m})\end{array}$ & $\begin{array}{c}\text { Left preconditioned } \\
\text { GMRES }(\mathrm{m})\end{array}$ & $\begin{array}{c}\text { Right preconditioned } \\
\text { GMRES(m) }\end{array}$ \\
\hline Number of iterations & $/$ & $>1000$ & 159 & 165 \\
CPU time/s & 515 & $/$ & 83 & 84 \\
\hline
\end{tabular}
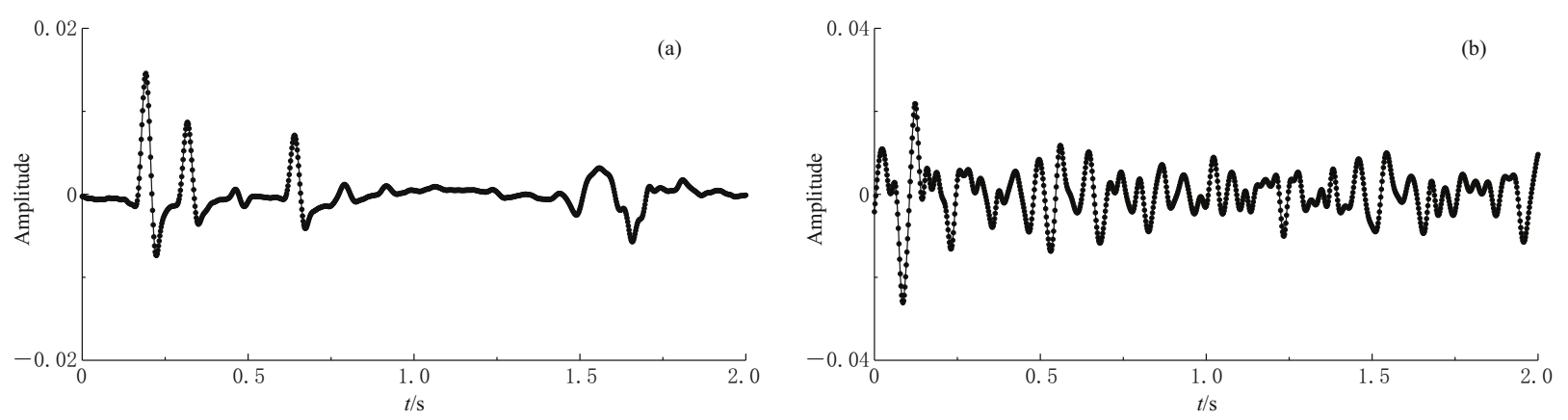

Figure 11 Comparison of the waveform recordings of mid-trace computed by the left diagonal preconditioned GMRES(m) method (dots) and the Gaussian elimination method (solid line) for random velocity perturbations of 0 (a) and 15 (b) percent in the near-surface layer, respectively.

with sediments), compared with the standard Gaussian elimination method. Then the method is tested by a heterogeneous multilayered model to show its effectiveness. The modeling results indicate that application of diagonal preconditioning improves convergence, with great savings on both computing time and memory requirements.

In summary, the preconditioned GMRES method significantly improves computational efficiency especially for large earth models and high frequencies, but with a faster convergence for the left diagonal preconditioning.

Acknowledgements We thank two anonymous reviewers for their constructive reviews and valuable comments. The research was supported by the 
National Natural Science Foundation of China (Nos. 41130418 and 40925013), and the National Basic Research Program (973 Program) (No. 2009CB219403).

\section{References}

Abramowitz M and Stegun I A (1968). Handbook of mathematical functions. Dover Publ. Inc, New York, 358-364.

Bakamjian B (1992). Boundary integrals: An efficient method for modeling seismic wave propagation in 3-D. 62th Ann Internat Mtg Soc Expl Geophys. New Orleans, USA, Expanded Abstracts, 1 232-1 235.

Bouchon M (1993). A numerical simulation of the acoustic and elastic wavefields radiated by a source on a fluidfilled borehole embedded in a layered medium. Geophysics 58: 475-481.

Campillo M (1987). Modeling of SH-wave propagation in an irregularly layered medium: Application to seismic profiles near a dome. Geophys Prospect 35: 236-249.

Cao P and Macaskill C (1995). Iterative techniques for rough surface scattering problems. Wave Motion 21: 209-229.

Chen G and Zhou H (1994). Boundary element modeling of nondissipative and dissipative waves. Geophysics 59: 113-118.

Dong W J, Bouchon M and Toksöz M N (1995). Borehole seismic-source radiation in layered isotropic and anisotropic media: Boundary element modeling. Geophysics 60: 735-747.

Fu L Y (1996). 3-D boundary element seismic modeling in complex geology. 66th Ann Internat Mtg Soc Expl Geophys. Denver, CO, USA, Expanded Abstracts, 1 239-1 242.

Fu L Y and Mu Y G (1994). Boundary element method for elastic wave forward modeling. Acta Geophysica Sinica 37: 521-529 (in Chinese with English abstract).

Fu L Y (2003). Numerical study of generalized LipmannSchwinger integral equation including surface topography. Geophysics 68: 665-671.

Liu E, Dobson A, Pan D M and Yang D H (2008a). The matrix formulation of boundary integral modeling of elastic wave propagation in $2 \mathrm{D}$ multi-layered media with irregular interfaces. J Comput Acous 16(3): 381-396.

Liu E, Zhang Z, Yue J and Dobson A (2008b). Boundary integral modeling of elastic wave propagation in multilayered 2D media with irregular interfaces. Communications in Computational Physics 3(1): 52-62.

Ma Z H (2005). Handbook of Modern Applied Mathematics: Calculation and Numerical Analysis. Tsinghua University Press, Beijing, 404-411 (in Chinese).

Pao Y H and Varatharajulu V (1976). Huygens' principle, radiation conditions and integral formulas for the scattering of elastic waves. J Acous Soc Amer 59: 1 361-1 370.

Saad Y and Schultz M H (1986). GMRES: A generalized minimal residual algorithm for solving nonsymmetric linear systems. SIAM J Sci Stat Comput 7(3): 856-869.

Wei B J and Liu Q H (2007a). Weak-form BCGS-FFT algorithm for volume integral equations in stratified medium. Journal of China University of Petroleum 31(1): 49-55 (in Chinese with English abstract).

Wei B J and Liu Q H (2007b). Fast algorithm for simulating 3-D electromagnetic inverse scattering in horizongtally stratified medium via DTA. Chinese Journal of Geophysics 50(5): 1 595-1 605 (in Chinese with English abstract).

Xia H M, Sun Y F and Song K H (2004). Application of GMRES to electromagnetic scattering problems from threedimensional dielectric scatterer. Journal of Anhui University Natural Science Edition 28(4): 47-50 (in Chinese with English abstract).

Xu S Z (1995). BEM in Geophysics. Science Press, Beijing, 34-39 (in Chinese).

Yu G X, Fu L Y and Yao Z X (2010). Comparison of different BEM+Born series modeling schemes for wave propagation in complex geologic structures. Geophysics $\mathbf{7 5}(3)$ : $\mathrm{T} 71-\mathrm{T} 82$.

Zhou H X and Hong W (2002). Improvement of MoM-CGFFT scheme for EM scattering from an infinite dielectric cylinder. Journal of Southeast University (Natural Science Edition) 32(2): 156-160 (in Chinese with English abstract). 